\title{
Affect conditioning associated with the onset and termination of electric shock
}

BERT FORRIN

SCARBOROUGH COLLEGE, UNIVERSITY OF TORONTO

The results of this study are consistent with the position that fear is more readily conditioned to a cue paired with the onset rather than the offset of a painful event. No evidence was found for the presumed secondary reinforcing properties of a cue associated with shock termination.

It is generally agreed that a stimulus paired with shock-induced pain acquires the capacity to elicit fear. There is, however, no unanimity of opinion regarding the conditions necessary for the learning of the fear reaction. Mowrer (1960) maintains that the association between an initially neutral cue and fear is formed solely on the basis of the repeated contiguity of the cue with the onset of pain. From a strict reinforcement theory, on the other hand, it follows that the reduction in drive afforded by the offset of the painful event is critical for the acquisition of fear (cf. Miller, 1951). The primary purpose of the present study was to examine the relative merits of these alternative views. A secondary objective was to explore the possibility that there is conditioned to a cue associated with shock termination not fear but "hope"-an affective state with reward value.

\section{Method}

The Ss were 180 experimentally naive, male albino rats, approximately 90 days old. For 7 days prior to the experiment proper, Ss were maintained on a $23 \mathrm{hr}$. food deprivation schedule and habituated to handling.

A 3-stage experimental procedure was employed with 1 day devoted to each stage.

1. Training. Ss, all $23 \mathrm{hr}$. hungry, were trained in a modified Skinner-box, 28 by 10 by 6 in., painted flat black. Depression of a food-trough located at one end of the chamber granted access to food pellets. To establish a base level of performance, Ss were permitted 30 reinforced trough presses. The base rate (in responses/min.) was determined for the 2-min. period preceding the final minute of training.

2. Affect conditioning. For one-half the Ss, affect conditioning was conducted in the chamber previously described; for the remainer, in a cylindrical compartment, 10 in. in diameter and 6 in. high, painted flat white. Each $S$ was clamped in position with its tail extending through an opening in the chamber wall; AC current (average intensity, .63 ma.) was delivered to electrodes fixed to S's tail. Behaviorally induced fluctuations in current intensity were thereby eliminated. The CS-a 2 sec., 4-cps interrupted buzzer-was presented in either an onset or an offset position. In the former case, the CS occurred 1 sec. before the onset of the 7-sec. shock and extended for $1 \mathrm{sec}$. into the shock period; in the latter, the cue was presented 1 sec. before and continued for $1 \mathrm{sec}$. after the termination of the US.

3. Testing. All Ss were tested in the chamber used for training. The trough-pressing response gave access to food and, simultaneously, produced the cue previously associated with shock. The interrupted buzzer continued to sound until the trough was released. Ss were given a 10-min. period of free responding timed from the first press. A test response rate was determined for the final $9 \mathrm{~min}$. of the test period to assess the effect of the experimental variables on the preshock level of performance.

The experimental design afforded an evaluation of the effects of four orthogonal variables: (1) temporal relation of cue to shock (onset or offset); (2) number of affect-conditioning trials $(1,4$, or 10); (3) strength of hunger drive during testing $(3,6$, or $23 \mathrm{hr}$. of food deprivation); and (4) similarity of the affect-conditioning and testing environments (identical or dissimilar). Five Ss were assigned at random to each of the 36 treatment combinations of the design.

\section{Results and Discussion}

The rationale for the inclusion of the onset-offset dimension is apparent. It permits a test of Mowrer's contention that the contiguity of a cue with a driveincrement is sufficient for the learning of fear. The number of affect-conditioning trials was varied to provide a specification of strength of fear independent of the crucial temporal parameter. The common assumption that strength of fear is indexed by performance decrement during testing cannot be granted a priori; it requires confirmation within the context of the present procedure. Strength of hunger drive during testing was investigated in recognition of the possibility that the effect of the feared cue might vary as a function of the strength of the approach tendency to the trough. Fear, for example, may serve to inhibit a weak response tendency but to facilitate a strong one. Finally, similarity of the affect-conditioning and testing environments was varied in "the attempt to institute the condition presumed by Mowrer to favor the demonstration of the secondary reward-or conditioned hope-properties of an offset cue. If the reward value of such a cue consists in its capacity to evoke a conditioned relaxation of fear, this value might be demonstrable only when fear is present during testing, that is, when the similarity of the affect-conditioning 
and testing chambers permits ready generalization of fear from the former to the latter.

Changes in response rate from training to testing are noted in Fig. 1. Analysis of differences in mean response rate revealed a statistically significant decrement in performance- $\mathrm{F}=185.54, \mathrm{df}=1 / 144, \mathrm{p}<.001$. The degree of response inhibition was an increasing function of the number of affect-conditioning trials$\mathrm{F}=6.58, \mathrm{df}=2 / 144, \mathrm{p}<.005$-and a decreasing function of the strength of hunger drive during testing $-\mathrm{F}=11.75$, $\mathrm{df}=2 / 144, \mathrm{p}<.001$. No significant interaction between the latter variables was observed. Of major concern, the performance decrement associated with the onset cue exceeded that associated with the offset cue$F=5.63, d f=1 / 144, p<.025$. The difference in the inhibitory effects of these cues was constant over all levels of the remaining variables.

These results must be interpreted with some caution. The significant decrement in performance is not attributable to the effect of the cues paired with shock alone; for two-thirds of the Ss, the hunger drive during testing was considerably reduced over that current during training. The absence of interactions among variables, however, suggests that the effects of onset and offset cues differed in degree but not

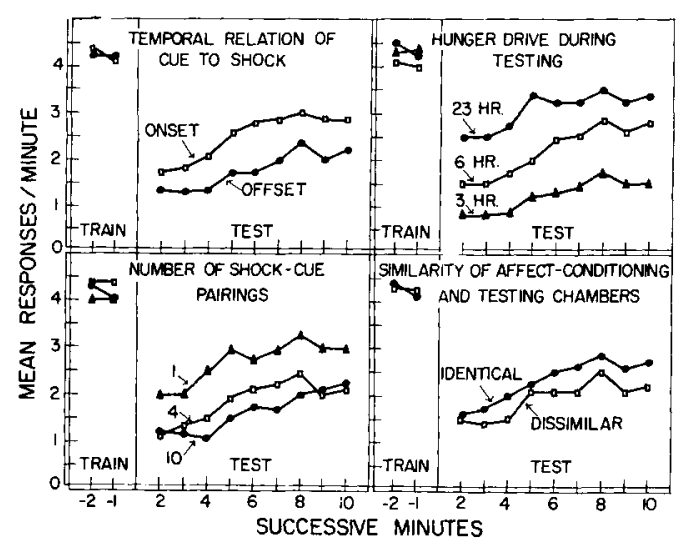

Fig. 1. Mean response rate over the $2 \mathrm{~min}$. preceding the final minute of training and over the final $9 \mathrm{~min}$. of testing plotted for each value of the four experimental variables. in kind. And the presence of a significant performance decrement- $\mathrm{F}=31.79$, $\mathrm{df}=1 / 48, \mathrm{p}<.001$-for those Ss both trained and tested while $23 \mathrm{hr}$. hungry bolsters the argument that the effect was an inhibitory one.

In sum, the findings support the conclusion that the strength of fear conditioned to a cue is reflected in the degree of inhibition of a response producing that cue. Since the decremental effect of the cue paired with shock onset exceeded that of the cue paired with shock termination, it may be inferred that fear was conditioned more strongly to the former. This inference is clearly consistent with Mowrer's position that the contiguity of a cue with the onset of a painful event is sufficient for the acquisition of the fear reaction. The force of the argument is somewhat diminished by the recognition of at least two potential sources of confounding: (a) during affect conditioning, the onset cue may have been more perceptible than the offset cue; and (b) skeletal responses of differential incompatibility with trough-pressing may have been conditioned to the two cues during the course of affect conditioning-say, "freezing" to the onset cue and "tugging forward" to the offset cue.

With regard to the second objective of the study, the results afford no support for the proposition that a cue in repeated contiguity with the cessation of a noxious state acquires reinforcing properties. The cue paired with shock offset failed to facilitate performance even when Ss were affect conditioned and tested in the same environment, a condition presumed to favor the demonstration of secondary reward value for an offset cue.

\section{References}

Miller, N. E. Learnable drives and rewards. In S. S. Stevens (Ed.), Handbook of experimental psychology. New York: Wiley, 1951. Pp. 435-472.

Mowrer, O. H. Learning theory and behavior. New York: Wiley, 1960 .

\section{Note}

1. Based on a dissertation submitted in partial fulfillment for the doctoral degree at the University of Michigan. This investigation was supported by a grant from the National Science Foundation. The author is indebted to Edward L. Walker and the late Russell A. Clark for their encouragement and assistance throughout the course of the study. 